\title{
Ön çapraz bağ yaralanması sonrası tedavi algoritması
}

\author{
Treatment algorithm after anterior cruciate ligament surgery
}

\author{
Gökhan Polat ${ }^{1}$, Sevan Sıvacıoğlu², Fatih Şentürk ${ }^{1}$
}

\author{
${ }^{1}$ İstanbul Üniversitesi İstanbul Tıp Fakültesi, Ortopedi ve Travmatoloji Anabilim Dalı, İstanbul \\ ${ }^{2}$ Acıbadem Üniversitesi Sağlık Bilimleri Fakültesi, Fizik Tedavi ve Rehabilitasyon Bölümü, İstanbul
}

\begin{abstract}
Ön çapraz bağ (ÖÇB) rüptürü; diz eklemi biyomekaniğini etkileyen oldukça sık görülen bir spor yaralanmasıdır. Bu yaralanmaların optimal tedavisi; klinik muayene ve objektif bulguların dışında, hastaların aktivite düzeyi ve beklentileri ile yakından iliş̧kilidir. Bu açıdan tedavide klinisyenlere rehber oluşturacak, kanıta dayalı olarak hazırlanmış ve değişmez bir tedavi rehberi ya da algoritması belirlenmesi oldukça güçtür. Hastalarda konservatif ya da cerrahi tedavi kararı; yaralanmanın tam kat ya da parsiyel olması, yaralanma zamanı, hastanın yaşı, eklem içi ek yaralanmaları, aktivite düzeyi, fonksiyonel beklenti ile diz ekleminin dejenerasyon miktarı gibi birçok faktör göz önünde bulundurularak verilmelidir. Orta-yaşlı sedanter bir yaşam süren izole ÖÇB yaralanmalarında, hastaların rehabilitasyon ve nöromusküler eğitimlerle konservatif izlemi denenebilir. Buna rağmen aktif bir yaşam süren ya da fonksiyonel beklentileri yüksek bireylerde, semptomatik instabilite varlığında normal diz eklemi biyomekaniğinin tekrar temin edilmesi amacıyla cerrahi tedavi uygulanmalıdır. Cerrahi tedavi zamanına; ÖÇB yaralanmasına eşlik eden bağ ya da menisküs yaralanması varlığı, hastanın sportif aktivite düzeyi ve beklentisine göre karar verilmelidir. Genel olarak ÖÇB yaralanması sonrası güvenli cerrahi tedavi; akut ağrılı dönemin geçirildiği, diz eklemi hareket açıklığının ve kuadriseps kas kontrolünün kazanıldığı dönemde uygulanmalıdır. Buna rağmen gecikmiş cerrahi ve diz ekleminin maruz kalacağı ek yaralanma riski nedeniyle cerrahi tedavinin ilk beş ay içerisinde yapılması önerilmektedir.
\end{abstract}

Anahtar sözcükler: ön çapraz bağ yaralanması; ek yaralanmalar; konservatif tedavi; cerrahi tedavi; cerrahi tedavide zamanlama

\begin{abstract}
Anterior cruciate ligament (ACL) rupture is a common sports injury that affects the biomechanics of the knee joint. The optimal treatment of this injury is not only related to the clinical examination and objective findings, but also closely related to the patient's activity level and expectations. So, it is very hard to determine an evidence-based, fixed clinical treatment guide or algorithm for clinicians. For the decision of conservative or surgical treatment of injury; multiple factors like partial or total rupture of ligament, injury time, patient's age, additional intraarticular injuries, patient's activity level and functional expectations, the degeneration level of joint should be considered. For middle-aged or old patients who has a sedentary life style, can be managed and followed conservatively with rehabilitation and neuromuscular training. However, patients with symptomatic instability who had an active life style or high demanding functional expectations, should be treated surgically in order to restore the normal knee biomechanics. The timing of surgery should be determined by consideration of additional injuries like meniscal or other ligaments, patient's sportive activity level and expectations. Generally, a safe surgical treatment after $A C L$ injury, should be performed after acute painful period of injury is ceased and knee range of motion with quadriceps muscle control is obtained. However, due to the delayed surgery and the risk of additional further injuries as a consequence of recurrent instability, the surgical treatment is recommended in the first 5 months of injury.
\end{abstract}

Key words: anterior cruciate ligament injury; additional injuries; conservative treatment; surgical treatment; timing of surgery

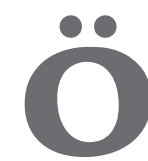

n çapraz bağ (ÖÇB) yaralanmaları sık görülen spor yaralanmalarından biridir ve diz eklemi bağ yaralanmalarının \%40-50'sini oluşturmaktadır. ${ }^{[1]}$ Genç-erişkin popülasyonda yapılan bir çalışmada ÖÇB'nin yıllık yaralanma insidansı 100.000'de 68,6 olarak belirtilmiştir. ${ }^{[2]}$ Yaralanmanın sıklığı, toplumların spor yapma alışkanlıklarındaki farklılıklara paralel olarak değişiklik göstermektedir. ${ }^{[3]}$ Ülkemizde bu konuda sağlıklı bir veri bulunmamaktadır.

Çalışmalarda yaralanmaların sıklıkla temassız şekilde (ani yön değiştirme, zıplama sonrası yere inerken gibi) gerçekleştiği bildirilmiştir. Biyomekanik çalışmalarda ÖÇB'nin üzerine binen en yüksek kuvvetin; diz eklemi tam ekstansiyona yakın pozisyonda iken diz

- İletişim adresi: Doç. Dr. Gökhan Polat, İstanbul Üniversitesi İstanbul Tıp Fakültesi, Ortopedi ve Travmatoloji Anabilim Dalı, İstanbul Tel: 0532 - 5406986 e-posta: gokhanpolat7@gmail.com

- Geliş tarihi: 24 Nisan $2020 \quad$ Kabul tarihi: 29 Nisan 2020 
üzerine tibianın iç rotasyon ve anterior translasyonuyla birlikte etkiyen valgus kuvveti ile oluştuğu gösterilmiştir. ${ }^{[4]}$ ÖÇB yaralanmaları futbol, basketbol ve kayak gibi spor dallarında daha sık görülmektedir. Yapılan epidemiyolojik çalışmalarda, ÖÇB yaralanmaları erkeklerde daha sık görülmektedir. Buna rağmen kadın cinsiyette; artmış $\mathrm{Q}$ açısı, posterior tibial slope (eğim), daha dar bir notch (çentik) ve bağ kesit alanı gibi risk faktörlerine bağlı olarak, ÖÇB yaralanma riskinin 2-8 kata kadar artmış olduğu bildirilmiştir. ÖÇB yaralanmaları; yüksek enerjili çoklu bağ yaralanmasının bir komponenti olarak ya da tek bir bağ yaralanması olarak görülebilmektedir. ${ }^{[5,6]}$ Literatürde ÖÇB yaralanması sonrasında, tedavide; konservatif tedavi, primer ÖÇB tamiri, parsiyel ÖÇB rekonstrüksiyonu ya da augmentasyonu, ÖÇB rekonstrüksiyonu, ÖÇB rekonstrüksiyonuna ek anterolateral ligament rekonstrüksiyonu ya da yüksek (proksimal) tibia osteotomisi gibi birçok tedavi tanımlanmış ve uygulanmaktadır. ${ }^{[7]}$

Oldukça sık karşılaşılan bu yaralanma için tedavide klinisyenlere rehber oluşturacak, kanıta dayalı olarak hazırlanmış, değişmez bir tedavi rehberi ya da algoritması belirlenmesi oldukça güçtür. Bunun en büyük nedeni klinik başarıyı etkileyen birçok değişkenin varlığı ve tedavi sonucuna etkisidir. ${ }^{[8]}$ Bunun dışında klinisyenler, doğru tedavi ve hastada tatminkâr sonuç elde etmek için klinik muayeneleri dışında; hastaların tercihleri, beklentileri ve bu yöndeki isteklerini de göz önünde bulundurmak durumundadır. ÖÇB yaralanmaları sonrası tedavi kararında; yaralanmanın tam kat ya da parsiyel olması, yaralanma zamanı, hastanın yaşı, eklem içi ek yaralanmalar, aktivite düzeyi, fonksiyonel beklenti, diz ekleminin dejenerasyon miktarı gibi birçok faktör göz önünde bulundurularak tedavi planlanmalıdır. ${ }^{[7,9]}$

\section{AKUT YARALANMA SONRASI ERIENN DÖNEM TEDAVi}

ÖÇB yaralanması sonrasında hastalar, sıklıkla akut hemartroz ve ağrı ile kliniğe başvururlar. Bu nedenle fizik muayene optimal şartlarda yapılamayabilir. Akut yaralanma sonrası hastaların tedavi planlamasında; yaralanmanın izole bir yaralanma mı, kombine bir yaralanmanın bir parçası mı olduğunun tespiti ya da eşlik eden bir menisküs ya da kıkırdak patolojisinin varlığı radyolojik incelemelerle birlikte değerlendirilmelidir. ${ }^{[10]}$ İzole ÖÇB yaralanması tespit edilen hastalarda, akut dönemde eklemde oluşan hemartroz ve enflamasyonun gerilemesine yönelik olarak diz ekleminin kompresif bandajla istirahati, buz uygulaması ve anti-enflamatuvar tedavi uygulanmalıdır. Diz hareket açıkığının kaybedilmemesi ve ekstremitenin atrofiden korunması için basit hareket açıklığı ve kuadriseps güçlendirme egzersizleri başlanmalıdır.

\section{KONSERVATIF TEDAVi}

Tam kat ÖÇB yaralanması sonrası diz ekleminde gelişen instabilite ve biyomekanik değişim, eklem içi sekonder hasar ve dejeneratif eklem artriti gelişimi riskini artırmaktadır. ${ }^{[9,11]}$ Aktif bir yaşam süren genç hastalarda (<35 yaş) ve sporcularda ÖÇB yaralanması sonrası konservatif tedavi; beklenen yüksek fiziksel aktivite seviyesi, gelişmesi muhtemel sekonder instabilite ve potansiyel ek yaralanmalar nedeniyle önerilmemektedir. ${ }^{[11]}$

Konservatif tedavi, sedanter bir yaşam tarzı olan ve spor aktivitelerine geri dönmeyecek orta-yaşlı ya da yaşı hastalarda ve artrozu ilerlemiş kronik ÖÇB yetmezliği olan hastalarda bir tedavi seçeneği olabilir. Seçilmiş hasta grubunda, yaralanma sonrası uygulanacak rehabilitasyon programları ve nöromusküler eğitimler ile günlük fiziksel aktivitelere dönüş mümkün olabilir. ${ }^{[12,13]}$ Literatürde yapılan çalışmalarda değişken sonuçlar bildirilmekle birlikte ${ }^{[14]}$, konservatif tedavi önerilecek hasta grubunda yaş sınıından ziyade aktivite seviyesinin ve eklemin dejeneratif durumunun göz önünde bulundurulması önerilmektedir. ${ }^{[15,16]}$

\section{KOMBINE BAĞ YARALANMALARI}

ÖÇB yaralanmaları, özellikle yüksek enerjili yaralanmalarda kombine bağ yaralanmasının bir komponenti olarak görülebilir. Diz çıkığı muadili (Schenk Tip 3 ve üzeri) yaralanmalarda, diğer yaralanan yapılarla birlikte ÖÇB yaralanmasının da cerrahi olarak tedavi edilmesi gereklidir. ${ }^{[16]}$

İç yan bağ (IYY) ve ÖÇB yaralanmalarında, medial tarafın yaralanma miktarına göre cerrahi tedavi ve zamanlamaya karar verilir. Grade (Evre) 1 ve 2 iYB yaralanması olan hastalarda, medial tarafin konservatif tedavisi sonrasında ÖÇB rekonstrüksiyonu uygulanabilir. Grade 3 IYB yaralanması ve ÖÇB yaralanması olan hastalarda ise tartışmalar sürse de yüksek aktivite beklentili hastalarda medial tarafın akut tamiri (ilk üç hafta) ve eş zamanlı ÖÇB rekonstrüksiyonu öne çıkmaktadır. ${ }^{[17]}$ Dizilim kusuru olmayan düşük beklentili orta-yaşlı hastalarda medial taraf Grade 3 yaralanmalar, mediolateral rigid (sert) destek sağlayan breysler (ateller) ile 3-4 hafta boyunca konservatif olarak izlenebilir. Hastanın klinik kontrollerinde mediolateral stabilite sağlanabilirse, hastanın beklentisine ve klinik muayenesine göre hasta rehabilitasyon ile konservatif takibe alınabilir. Aksi durumda hastanın cerrahi tedavisi ön plana çıkmaktadır. ${ }^{[17]}$

Lateral tarafın primer iyileşme kapasitesi, biyomekanik olarak bu bölgeye binen distraktif yükler nedeniyle, medial tarafa göre daha zayıftır. Bu açıdan Grade 1 ve 2 lateral taraf yaralanması olan hastalar, 
ekstremitenin yükten kurtarılması ile birlikte mediolateral rijit breys (atel) ile konservatif olarak izlenir. Lateral tarafın iyileşmesi sonrasında ÖÇB rekonstrüksiyonu uygulanır. ${ }^{[18]}$ Eşlik eden lateral taraf Grade 3 yaralanmalarda ise hastaların erken dönemde kombine cerrahi ile tedavisi önerilmektedir. ${ }^{[18]}$

\section{EŞLIK EDEN AKUT MENISKÜS YIRTIKLARI}

Menisküs yaralanmaları ÖÇB yaralanmalarına en sık olarak eşlik eden patolojilerdir. Hastalarda akut yaralanmalarda lateral menisküs (daha sık) ve medial menisküs yaralanmaları gelişebilmektedir. Deplase kova sapı yırtık gibi mekanik blok oluşturan menisküs patolojilerinde, cerrahi tedavi mümkün olduğunca erken dönemde yapılmalıdır. Mekanik semptom yaratmayan menisküs yırtıklarının tedavisinde ise ÖÇB rekonstrüksiyonu için planlanan standart tedavi protokolü uygulanabilir. Menisküs tamiri ile ilgili literatür, ÖÇB rekonstrüksiyonu ile birlikte uygulanan menisküs tamiri sonuçları oldukça başarılı olduğunu göstermektedir. ${ }^{[19]} \mathrm{Bu}$ nedenle bu hasta grubunda menisküsler mümkün olduğunca korunmalı ve tamir ön planda düşünülmelidir. Bununla birlikte menisküs kök yırtıkları ya da ramp lezyonu gibi instabiliteye sekonder olarak gelişen yırtıkların tamir edilmesinin, elde edilen klinik sonuçların başarısını artırdığını bildiren yeni çalışmalar da mevcuttur. ${ }^{[20]}$

\section{CERRAHI TEDAVIDE ZAMANLAMA}

ÖÇB yaralanmaları sonrasında, tedavinin amacı hastaların yaralanma öncesindeki fiziksel kapasitelerine geri dönmesini sağlayacak, yeterli stabiliteye ve hareket açıklığına sahip diz ekleminin tekrar temin edilmesidir. Bu açıdan aktif bir yaşam süren ve artrozik bir ekleme sahip olmayan tüm bireylerde, semptomatik instabilite varlığında normal diz eklemi biyomekaniğini tekrar elde etmek amaciyla ÖÇB rekonstrüksiyonu uygulanmalıdır. ${ }^{[21,22]}$

İzole ÖÇB yaralanması (ek yaralanmaları olmayan) sonrasında cerrahi tedavide zamanlama tartışmalı bir konudur. Erken cerrahide artmış artrofibroz riski bildiren çalışmalara rağmen, son yıllarda yapılan çalışmalarda erken cerrahi ile geç cerrahi yapılan gruplar arasında fark olmadığını bildiren çalışmalar da yayımlanmıştır. ${ }^{[23,24]}$ Genel olarak ÖÇB yaralanması sonrasında; akut enflamasyon ve ağrılı dönemin geçirildiği, diz ekleminde tam hareket açıklığının ve kuadriseps kas kontrolünün kazanıldığı dönemde cerrahi tedavi uygulanması önerilmektedir. ${ }^{[21]}$ Buna rağmen gecikmiş cerrahi ve diz ekleminin maruz kalacağı ek yaralanma riski nedeniyle cerrahi tedavinin ilk beş ay içerisinde yapılması önerilmektedir. ${ }^{[22]}$

\section{ARTROZLU EKLEMLERDE GELIŞEN ÖÇB YARALANMALARI}

Artrozlu diz eklemi olan hastaların ÖÇB yaralanmaları sonrasında ya da uzun süreli ihmal edilmiş ÖÇB yetmezliğine bağlı artrozu ve instabilitesi olan hastalarda ideal tedavi ile ilişkili tartışmalar halen devam etmektedir. Bu konudaki klinik yaklaşımda, hastaya özgü bir değerlendirme yapılması ve hastanın klinik şikayetlerinin (ağrı varlığı, ağrının karakteri, dizde boşalma gibi instabilite epizodları vb.) iyi sorgulanması gereklidir. Literatürde, instabilite şikâyetlerinin ön planda olduğu hastalarda ÖÇB rekonstrüksiyonu sonrası, stabilitenin sağlandığı ve ağrının azaldığını bildiren çalışmalar mevcuttur. Buna rağmen bu hastaların eklem dejenerasyonunun ameliyat sonrası dönemde ilerleyebildiği, istirahat ağrılarının devam ettiği ve bu açıdan yüksek aktivite düzeyi gerektiren işlerden ve sporlardan uzak kalmaları gerektiği belirtilmektedir. ${ }^{[25,26]}$ Koronal plan deformiteleri ve buna bağlı düşük-orta evre artrozu olan hastalarda, stabilitenin tekrar temin edilmesi ve diz ekleminin biyolojik sağ-kalımının uzatılması için ÖÇB rekonstrüksiyonuna ek olarak eşzamanlı yüksek (proksimal) tibial osteotomi ile dizilim düzeltilmelidir. ${ }^{[27]}$

\section{PEDIATRIK HASTALAR}

İskelet matüritesi tamamlanmamış çocuk ve adolesan hasta grubunda, ÖÇB yaralanmaları tedavisi halen tartışmalı bir konudur. Bu hasta grubunda tedavi kararında iskelet matüritesinin değerlendirilmesi esastır. Buna göre klinik instabilite gelişen hastalarda cerrahi tedavide; ekstra-artiküler rekonstrüksiyonlar, primer tamir, kısmi ya da tamamı epifiziyel rekonstrüksiyonlar ve transepifiziyel rekonstrüksiyonlar tanımlanmıştır. ${ }^{[28]}$

Rekonstrüksiyonlar sonrasında; olası fiz hasarı ve deformite gelişimi, ekstremitenin uzaması sırasında rekonstrükte edilen bağın büyüme ile değişimi ve görece diğer yaş gruplarına kıyasla daha düşük klinik başarı nedeniyle bu yaş gurunda rutin cerrahi tedavi sorgulanmaktadır. Buna rağmen tedavisiz bırakılan pediatrik hastaların, aktivite kısıtlamasının başarılı olamaması, gelişen sekonder menisküs ve kıkırdak yaralanmaları da konservatif izlem sırasında karşılaşılan önemli sorunlardır. İskelet matüritesini tamamlamamış hastaların tedavisiyle ilişkili literatürün genel değerlendirmesinde; Tanner Evre 4 ve 5 gelişime sahip adolesanlar da erişkinlere benzer transepifiziyel rekonstrüksiyonların, daha düşük gelişime sahip sporcularda ise fizi koruyan cerrahi rekonstrüksiyon yöntemlerinin uygulanmasının güvenli olduğu söylenebilir. ${ }^{[28,29]}$ Tedavi, ailenin bilgilendirilmesi ve iş birliğinde yürütülmelidir. 


\section{ERIŞKIN HASTALARDA PRIMER TAMIR}

ÖÇB primer tamiri; yaralanan bağ dokusunun kalitesi, bağın sınırlı iyileşme kabiliyeti ve eklem hareketleri esnasında tamir edilen doku üzerine binen ciddi mekanik yükler nedeniyle şüphe ile yaklaşılan bir tedavidir. Buna rağmen özellikle femoral yapışma yerine yakın akut yaralanmalarda (ilk üç hafta), remnant (artık, kalıntı) bağ dokusunun içerisinden geçirilen yüksek dayanıklılığa sahip sütür materyalinin kemik çapaları ya da transosseöz tüneller yardımıyla femoral yapışma yerine tekrar tespiti ile primer tamirler uygulanabilmektedir. Son yıllarda dinamik interligamenter stabilizasyon tekniğinin ortaya çıkmasıyla primer tamir daha popülarize olmuştur. Henüz literatürde ÖÇB yaralanmaları sonrası rutin uygulama için yeterli bilimsel kanıt bulunmamaktadır. ${ }^{[30]}$

\section{SONUÇ VE TEDAVI ALGORITMASI}

ÖÇB rüptürü; diz eklemi biyomekaniğini etkileyen oldukça sık görülen bir spor yaralanmasıdır. Sıklıkla genç-aktif hastaların etkilenmesine rağmen, zaman zaman daha yaşlı ve sedanter hastalar da etkilenebilmektedir. Bu yaralanmaların optimal tedavisi; klinik muayene ve objektif bulguların dışında, hastaların aktivite düzeyi ve beklentileri ile yakından ilişkilidir. Bu açıdan tedavide klinisyenlere rehber oluşturacak, kanıta dayalı olarak hazırlanmış, değişmez bir tedavi rehberi ya da algoritması belirlenmesi oldukça güçtür. Bunun en büyük nedeni klinik başarıyı etkileyen birçok değişkenin varlığı ve tedavi sonucuna etkisidir. ${ }^{[8]}$ Bunun dışında klinisyenler, doğru tedavi ve hastada tatminkâr sonuç elde etmek için klinik muayeneleri dışında; hastaların tercihleri, beklentileri ve bu yöndeki isteklerini de göz önünde bulundurmak durumundadır.

Klinisyen; hastada klinik olarak instabilite varlığını ayrıntılı bir hikâye ve fizik muayene ile ortaya koymalı, ideal radyolojik incelemelerle ÖÇB yaralanmasını ve ek lezyonları görüntülemelidir. Tedavide ise; yaralanmanın tam kat ya da parsiyel olması, yaralanma zamanı, hastanın yaşı, eklem içi ek yaralanmaları, aktivite düzeyi, fonksiyonel beklenti, diz ekleminin dejenerasyon miktarı gibi birçok faktör göz önünde bulundurularak planlama yapılmalıdır. ${ }^{[7,9]}$

\section{KAYNAKLAR}

1. Hirshman HP, Daniel DM, Miyasaka K. The fate of unoperated knee ligament injuries. In: Daniel D, editor. Knee Ligaments: Structure, Function, Injury, and Repair. New York: Raven Press; 1990.

2. Sanders TL, Maradit Kremers H, Bryan AJ, Larson DR, Dahm DL, Levy BA, Stuart MJ, Krych AJ. Incidence of Anterior Cruciate Ligament Tears and Reconstruction: A 21-Year Population-Based Study. Am J Sports Med 2016;44(6):1502-7. Crossref
3. Prentice $H A$, Lind $M$, Mouton C, Persson A, Magnusson H, Gabr A, Seil R, Engebretsen L, Samuelsson K, Karlsson J, Forssblad M, Haddad FS, Spalding T, Funahashi TT, Paxton LW, Maletis GB. Patient demographic and surgical characteristics in anterior cruciate ligament reconstruction: a description of registries from six countries. Br J Sports Med 2018;52(11):716-22. Crossref

4. Oh YK, Lipps DB, Ashton-Miller JA, Wojtys EM. What strains the anterior cruciate ligament during a pivot landing? Am J Sports Med 2012;40(3):574-83. Crossref

5. Sutton KM, Bullock JM. Anterior cruciate ligament rupture: differences between males and females. J Am Acad Orthop Surg 2013;21(1):41-50. Crossref

6. Cain EL Jr, Fleisig GS, Ponce BA, Boohaker HA, George MP, McGwin G Jr, Andrews JR, Lemak LJ, Clancy WG Jr, Dugas JR. Variables Associated with Chondral and Meniscal Injuries in Anterior Cruciate Ligament Surgery. J Knee Surg 2017;30(7):659-67. Crossref

7. Cabitza F, Ragone V, Arrigoni P, Karlsson J, Randelli P. Management of knee injuries: consensus-based indications from a large community of orthopaedic surgeons. Knee Surg Sports Traumatol Arthrosc 2013;21(3):708-19. Crossref

8. Yabroudi MA, Björnsson H, Lynch AD, Muller B, Samuelsson K, Tarabichi M, Karlsson J, Fu FH, Harner CD, Irrgang JJ. Predictors of Revision Surgery After Primary Anterior Cruciate Ligament Reconstruction. Orthop J Sports Med 2016;4(9):2325967116666039. Crossref

9. Smith TO, Postle K, Penny F, McNamara I, Mann CJV. Is reconstruction the best management strategy for anterior cruciate ligament rupture? A systematic review and metaanalysis comparing anterior cruciate ligament reconstruction versus non-operative treatment. Knee 2014;21(2):462-70. Crossref

10. Benjaminse A, Gokeler A, van der Schans CP. Clinical diagnosis of an anterior cruciate ligament rupture: a metaanalysis. J Orthop Sports Phys Ther 2006;36(5):267-88. Crossref

11. Nebelung $\mathbf{W}$, Wuschech $\mathrm{H}$. Thirty-five years of follow-up of anterior cruciate ligament-deficient knees in high-level athletes. Arthroscopy 2005;21(6):696-702. Crossref

12. Trees $\mathrm{AH}$, Howe TE, Dixon J, White L. Exercise for treating isolated anterior cruciate ligament injuries in adults. Cochrane Database Syst Rev 2005:CD005316. Crossref

13. Ahn JH, Chang MJ, Lee YS, Koh KH, Park YS, Eun SS. Nonoperative treatment of ACL rupture with mild instability. Arch Orthop Trauma Surg 2010;130(8):1001-6. Crossref

14. Delincé P, Ghafil D. Anterior cruciate ligament tears: conservative or surgical treatment? Knee Surg Sports Traumatol Arthrosc 2013;21(7):1706-7. Crossref

15. Monk AP, Davies LJ, Hopewell S, Harris K, Beard DJ, Price AJ. Surgical versus conservative interventions for treating anterior cruciate ligament injuries. Cochrane Database Syst Rev 2016;4:CD011166. Crossref

16. Marks $\mathrm{PH}$, Harner $\mathrm{CD}$. The anterior cruciate ligament in the multiple ligament-injured knee. Clin Sports Med 1993;12(4):825-38

17. Stannard JP, Bauer KL. Current concepts in knee dislocations: PCL, ACL, and medial sided injuries. J Knee Surg 2012;25(4):287-94. Crossref

18. Dean RS, LaPrade RF. ACL and Posterolateral Corner Injuries. Curr Rev Musculoskelet Med 2020;13(1):123-32. Crossref 
19. De Girolamo L, Galliera E, Volpi P, Denti M, Dogliotti G, Quaglia A, Cabitza P, Corsi Romanelli MM, Randelli P. Why menisci show higher healing rate when repaired during $A C L$ reconstruction? Growth factors release can be the explanation. Knee Surg Sports Traumatol Arthrosc 2015;23(1):90-6. Crossref

20. Mouton C, Magosch A, Pape D, Hoffmann A, Nührenbörger C, Seil R. Ramp lesions of the medial meniscus are associated with a higher grade of dynamic rotatory laxity in ACL-injured patients in comparison to patients with an isolated injury. Knee Surg Sports Traumatol Arthrosc 2020;28(4):1023-8. Crossref

21. Meuffels DE, Poldervaart MT, Diercks RL, Fievez AWFM, Patt TW, Hart CP, Hammacher ER, van der Meer F, Goedhart EA, Lenssen AF, Muller-Ploeger SB, Pols MA, Saris DBF. Guideline on anterior cruciate ligament injury. Acta Orthop 2012;83(4):379-86. Crossref

22. Shea KG, Carey JL. Management of anterior cruciate ligament injuries: evidence-based guideline. J Am Acad Orthop Surg 2015;23(5):e1-5. Crossref

23. Shelbourne KD, Wilckens JH, Mollabashy A, DeCarlo M. Arthrofibrosis in acute anterior cruciate ligament reconstruction. The effect of timing of reconstruction and rehabilitation. Am J Sports Med 1991;19(4):332-6. Crossref

24. Smith TO, Davies L, Hing CB. Early versus delayed surgery for anterior cruciate ligament reconstruction: a systematic review and meta-analysis. Knee Surg Sports Traumatol Arthrose 2010;18(3):304-11. Crossref
25. Shelbourne KD, Wilckens JH. Intraarticular anterior cruciate ligament reconstruction in the symptomatic arthritic knee. Am J Sports Med 199;21(5):685-9. Crossref

26. Kim SJ, Park KH, Kim SH, Kim SG, Chun YM. Anterior cruciate ligament reconstruction improves activity-induced pain in comparison with pain at rest in middle-aged patients with significant cartilage degeneration. Am J Sports Med 2010;38(7):1343-8. Crossref

27. Stride D, Wang J, Horner NS, Alolabi B, Khanna V, Khan $M$. Indications and outcomes of simultaneous high tibial osteotomy and $\mathrm{ACL}$ reconstruction. Knee Surg Sports Traumatol Arthrosc 2019;27(4):1320-31. Crossref

28. Fabricant PD, Jones KJ, Delos D, Cordasco FA, Marx RG, Pearle AD, Warren RF, Green DW. Reconstruction of the anterior cruciate ligament in the skeletally immature athlete: a review of current concepts: AAOS exhibit selection. J Bone Joint Surg Am 2013;95(5):e28. https://journals.Iww.com/ jbjsjournal/Abstract/2013/03060/Reconstruction_of_the_ Anterior_Cruciate_Ligament.17.aspx

29. Joseph SM, Huleatt JB, Vogel-Abernathie LA, Pace JL. Treatment of ACL Tears in the Skeletally Immature Patient. Sports Med Arthrosc Rev 2018;26(4):153-6. Crossref

30. Papalia R, Torre G, Papalia G, Campi S, Maffulli N, Denaro V. Arthroscopic primary repair of the anterior cruciate ligament in adults: a systematic review. Br Med Bull 2019;131(1):2942. Crossref 